\title{
Quantum state transformation and general design scheme on teleportation protocols
}

\author{
JIANG Min $^{1 *}$, HUANG Xu ${ }^{1}$, ZHOU LiuLie ${ }^{1}$, ZHOU YiMing $^{2} \&$ ZENG Jia ${ }^{3}$ \\ ${ }^{1}$ School of Electronics \& Information Engineering, Soochow University, Suzhou 215006, China; \\ ${ }^{2}$ Beijing Institute of Space Science and Technology Information, Beijing 100086, China; \\ ${ }^{3}$ The 208th Institute of the Second Research Academy, CASIC, Beijing 100854, China
}

Received October 21, 2011; accepted December 29, 2011; published online April 24, 2012

\begin{abstract}
We present a scheme for probabilistic transformation of special quantum states assisted by auxiliary qubits. In our scheme, if quantum states can be rewritten in a particular form, it is possible to transform such states into other states using lowerdimensional unitary operations that can be more easily realized in physical experiments. Furthermore, as an important application, we propose a generalized scheme that helps construct faithful quantum channels via various probabilistic channels when considering the existence of nonmaximally-entangled states.
\end{abstract}

probabilistic teleportation, auxiliary particle, bell-state measurement, state transformation

Citation: Jiang M, Huang X, Zhou L L, et al. Quantum state transformation and general design scheme on teleportation protocols. Chin Sci Bull, 2012, 57: 22472251, doi: 10.1007/s11434-012-5163-6

Over the past decade or so, there have been dramatic developments in quantum information processing from both theoretical and experimental aspects [1-3]. Quantum control plays a key role in quantum information technology as quantum gates are not hardwired as in traditional chips, but instead involve sophisticated manipulations of quantum systems [1-11]. In particular, if information is represented in quantum mechanical states, quantum information processing is essentially the manipulation of these quantum states. However, controlling a quantum system at a desired level is entirely different to control classical counterparts and raises an entirely new set of issues in control theory. Note that a teleportation-based strategy can accomplish some tasks such as distant feedback of a coherent quantum state that usually cannot be completed by coherent controls with classical feedback in a similar manner as Markovian quantum feedback [4]. In detail, quantum teleportation can be used to construct feedback controllers with the output from the quantum system to be controlled fed back into an actuator via teleportation to alter the dynamics of the system

*Corresponding author (email: jiangmin03@gmail.com)
[5]. In that way, quantum teleportation performs distant transmissions that can be viewed as part of a quantum feedback control loop. Alternatively, we can consider the implementation of an arbitrary unitary gate upon a distant quantum system. This teleportation of a quantum unitary gate can be viewed as a quantum remote control $[9,10]$. Therefore, a detailed study of quantum teleportation would greatly contribute to a better understanding of quantum control. Due to its potential application, quantum teleportation has drawn the attention of many researchers [12-28]. Moving onwards from teleportation of a one-particle or two-particle unknown state, some researchers have focused on the teleportation of multi-particle states [29-33], controlled teleportation $[34,35]$, and probabilistic teleportation [36-40]. Dong et al. [40] considered probabilistic and controlled quantum teleportation given that the quantum channel was not in the form of an exact GHZ state. By far, much of the many probabilistic or deterministic teleportation protocols is based on the nonmaximally-entangled states. In our previous work [32,33], we developed a scheme for establishing a faithful quantum channel for both indirect and direct teleportation that could be applied in a teleportation 
network where intermediate relay agents exist between a sender and a receiver.

In most probabilistic teleportation protocols, various unitary gates are performed on some qubits and one auxiliary qubit held by a sender or a receiver involved in the implementation of teleportation circuit. However, no unified scheme has been reported to explain the cause and effect of the high-dimensional unitary operations. In other words, the general problem remains still open to construct teleportation protocols via various quantum states obtained in the physical realization.

In this paper, we present a general construction of teleportation protocol via various quantum states assisted by auxiliary qubits. First, we consider the generalized form of a special class of quantum state and present a scheme to implement probabilistic transformation with minimal resources. Next, we apply our method and theoretical analysis to further propose a general scheme for teleportation protocols based on various nonmaximally-entangled channels. Finally, we give a brief discussion and summary.

\section{State probabilistic transformation assisted by ancillary particles}

Let us start with one multi-particle state, assumed normalized, that can be expanded in the following manner:

$$
|\varphi\rangle_{A_{1} A_{2} \cdots A_{n} B_{1} B_{2} \cdots B_{m}}=\sum_{i} a_{i}\left|\left\{j_{l}\right\}\right\rangle_{A_{1} A_{2} \cdots A_{n}}\left|\left\{t_{f}\right\}\right\rangle_{B_{1} B_{2} \cdots B_{m}},
$$

where $\quad\left|\left\{j_{l}\right\}\right\rangle=\left|j_{1} j_{2} \cdots j_{n}\right\rangle\left(j_{l} \in\{0,1\}(l=1,2, \cdots n)\right)$ denote the basis computational states of $n$ particles belonging to the particle set $\{A\}$ whereas $\left|\left\{t_{f}\right\}\right\rangle=\left|t_{1} t_{2} \cdots t_{m}\right\rangle\left(t_{f} \in\{0,1\}\right.$ $(l=1,2, \cdots m))$ denote the basis computational states of $m$ particles belonging to the particle set $\{B\}$. It should be noted that in stating (1) each basis computational state $\left|\left\{t_{f}\right\}\right\rangle_{B_{1} B_{2} \cdots B_{m}}=\left|t_{1} t_{2} \cdots t_{m}\right\rangle_{B_{1} B_{2} \cdots B_{m}}\left(t_{f} \in\{0,1\}(l=1,2, \cdots m)\right)$ can only be combined with at most one basis computational state $\left|\left\{j_{l}\right\}\right\rangle_{A_{1} A_{2} \cdots A_{n}}=\left|j_{1} j_{2} \cdots j_{n}\right\rangle_{A_{1} A_{2} \cdots A_{n}}\left(j_{l} \in\{0,1\}(l=1,2, \cdots n)\right)$.

With use of one auxiliary qubit and an $n+1$ dimensional unitary gate, we can obtain the following state with a certain probability:

$$
\left|\varphi^{\prime}\right\rangle_{A_{1} A_{2} \cdots A_{n} B_{1} B_{2} \cdots B_{m}}=\sum_{i} b_{i}\left|\left\{j_{l}\right\}\right\rangle_{A_{1} A_{2} \cdots A_{n}}\left|\left\{t_{f}\right\}\right\rangle_{B_{1} B_{2} \cdots B_{m}} .
$$

Here, as for $|\varphi\rangle_{A_{1} A_{2} \cdots A_{n} B_{1} B_{2} \cdots B_{m}}$ in eq. (1) above, $\left|\varphi^{\prime}\right\rangle_{A_{1} A_{2} \cdots A_{n} B_{1} B_{2} \cdots B_{m}}$ is normalized.

Proof. Assume $k_{i}=a_{i} / b_{i}$ and $k_{m}=\min \left\{k_{i}\right\}(i=0,2$, $\left.\cdots 2^{n-1}\right)$. The specific circuit implementation of the whole process is illustrated in Figure 1.

First, we introduce an ancillary particle $|0\rangle_{r}$ and perform the following unitary operation $U_{B_{1} B_{2} \cdots B_{n} r}$ on particles
$B_{i}(i=1,2, \ldots n)$ and the ancillary particle $r$ :

$$
U_{B_{1} B_{2} \cdots B_{n} r}=\left[\begin{array}{cccc}
U_{0} & & & \\
& U_{2} & & \\
& & \ddots & \\
& & & U_{2^{n-1}}
\end{array}\right] .
$$

Here

$$
U_{i}=\left[\begin{array}{cc}
\frac{k_{m}}{k_{i}} & \sqrt{1-\frac{k_{m}^{2}}{k_{i}^{2}}} \\
\sqrt{1-\frac{k_{m}^{2}}{k_{i}^{2}}} & -\frac{k_{m}}{k_{i}}
\end{array}\right] .
$$

Next, after performing this unitary operation, the state of particles $A_{1}, A_{2}, \cdots A_{n}, B_{1}, B_{2}, \cdots B_{m}$ and $r$ reads

$$
\begin{aligned}
& |\varphi\rangle_{A_{1} A_{2} \cdots A_{n} B_{1} B_{2} \cdots B_{m}} \\
= & k_{m} \sum_{i} b_{i}\left|\left\{j_{l}\right\}\right\rangle_{A_{1} A_{2} \cdots A_{n}}\left|t_{i}\right\rangle_{B_{1} B_{2} \cdots B_{m}}|0\rangle_{r} \\
& +\sum_{i} b_{i} \sqrt{k_{i}^{2}-k_{m}^{2}}\left|\left\{j_{l}\right\}\right\rangle_{A_{1} A_{2} \cdots A_{n}}\left|t_{i}\right\rangle_{B_{1} B_{2} \cdots B_{m}}|1\rangle_{r} .
\end{aligned}
$$

Finally, we perform a measurement on the state of the auxiliary particle $r$ spanned by the basis $\{|0\rangle,|1\rangle\}$. If the result is $|0\rangle_{r}$, then the state of particles $A_{1}, A_{2}$, $\cdots A_{n}, B_{1}, B_{2}, \cdots B_{m}$ will have been transformed to $\left|\varphi^{\prime}\right\rangle_{A_{1} A_{2} \cdots A_{n} B_{1} B_{2} \cdots B_{m}}$; i.e. the transformation is successfully realized. However, if the result is $|1\rangle_{r}$, then the transformation will have failed. The probability of successful transformation can be calculated as $\left|k_{m}\right|^{2}$.

Note that if the multi-particle state can be written in the form (1), the particle set $\left\{A_{i}\right\}(i=1,2, \ldots n)$ will be named as our particular particle set. Thus, it is essential to our scheme to find the particular particle set $\left\{A_{i}\right\}(i=1,2, \ldots n)$. Furthermore, the transformation between $|\varphi\rangle_{A_{1} A_{2} \cdots A_{n} B_{1} B_{2} \cdots B_{m}}$ and $\left|\varphi^{\prime}\right\rangle_{A_{1} A_{2} \cdots A_{n} B_{1} B_{2} \cdots B_{m}}$ can be regarded as the transformation between the following two states:

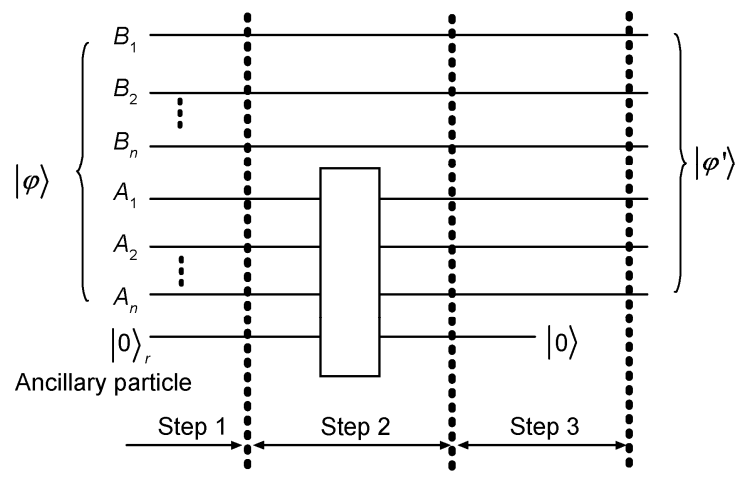

Figure 1 Circuit representation for the probabilistic transformation of quantum states assisted by an auxiliary qubit. 


$$
|\varphi\rangle_{A_{1} A_{2} \cdots A_{n}}^{*}=\sum_{i} a_{i}\left|\left\{j_{l}\right\}\right\rangle_{A_{1} A_{2} \cdots A_{n}},
$$

and

$$
\left|\varphi^{\prime}\right\rangle_{A_{1} A_{2} \cdots A_{n}}^{*}=\sum_{i} b_{i}\left|\left\{j_{l}\right\}\right\rangle_{A_{1} A_{2} \cdots A_{n}} .
$$

Consider the extreme case when, in the beginning, the particle set $\left\{A_{i}\right\}(i=1,2, \ldots n)$ represent all the particles comprising the joint system. In the following, we show that it is possible to transform the initial state to any target state composed of all or less than $n$ particles with a certain probability.

Based on the above formalism, we will give a brief description of the protocol in regard to the probabilistic transformation of quantum states as shown in Figure 1.

(1) Depending upon the relationship between initial and target states in the entangled joint system, pick the particular particle set $\left\{A_{i}\right\}(i=1,2, \ldots n)$;

(2) Next, according to the transformation relationship between the original and target states, import an auxiliary qubit, design a unitary gate operation and perform it on $\left\{A_{i}\right\}(i=1,2, \ldots n)$ and the auxiliary qubit;

(3) Finally, perform a von Neumann measurement on the auxiliary qubit. If the measurement outcome fulfills the requirement, the transformation has succeeded; otherwise, it has failed.

\section{Construction of the faithful channel via various quantum states}

Based on the analysis in the above section, we will further explore the scheme for construction of the faithful channel via various quantum states.

As a starting point, let us take a more concrete example [27] where the channel is in the following state:

$$
\begin{aligned}
|\varphi\rangle_{34567}= & \alpha|00000\rangle_{34567}+\beta|01100\rangle_{34567} \\
& +r|10011\rangle_{34567}+k|11111\rangle_{34567} .
\end{aligned}
$$

It can be written either as

$$
\begin{aligned}
|\varphi\rangle_{34567}= & \alpha|00\rangle_{34}|000\rangle_{567}+\beta|01\rangle_{34}|100\rangle_{567} \\
& +r|10\rangle_{34}|011\rangle_{567}+k|11\rangle_{34}|111\rangle_{567}
\end{aligned}
$$

or as

$$
\begin{aligned}
|\varphi\rangle_{34567}= & \alpha|00\rangle_{56}|000\rangle_{347}+\beta|10\rangle_{56}|010\rangle_{347} \\
& +r|01\rangle_{56}|101\rangle_{347}+k|11\rangle_{56}|111\rangle_{347} .
\end{aligned}
$$

That is to say, particle set $\{3,4\}$ or $\{5,6\}$ can be regarded as the particular particle set. Therefore, to obtain a faithful channel, either sender Alice holding the particle set $\{3,4\}$ or receiver Bob holding the particle set $\{3,4\}$ can take appropriate measurements to play the role of channel modulator. Finally, if we make use of the scheme proposed in Section 1 , then the initial system can with a certain probability be transformed into the following un-normalized form:

$$
\begin{aligned}
|\varphi\rangle_{34567}= & |00000\rangle_{34567}+|01100\rangle_{34567} \\
& +|10011\rangle_{34567}+|11111\rangle_{34567} \\
= & \left(|00\rangle_{36}+|11\rangle_{36}\right)\left(|00\rangle_{45}+|11\rangle_{45}\right)\left(|0\rangle_{7}+|1\rangle_{7}\right) \\
& +\left(|00\rangle_{36}-|11\rangle_{36}\right)\left(|00\rangle_{45}+|11\rangle_{45}\right)\left(|0\rangle_{7}-|1\rangle_{7}\right)
\end{aligned}
$$

signifying that a faithful channel has been established to implement either deterministic or controlled teleportation. Note that the above analysis gives a theoretical interpretation and is totally consistent with the probabilistic teleportation protocol in [34].

Similar theoretical analysis can also be applied to the inexact GHZ state form [22,35,36]:

$$
\begin{aligned}
|\mathrm{GHZ}\rangle_{123} & =\alpha|000\rangle_{123}+\beta|111\rangle_{123} \\
& =\alpha|0\rangle_{1}|00\rangle_{23}+\beta|1\rangle_{1}|11\rangle_{23},
\end{aligned}
$$

where the sender, receiver and controller possess particles 1 , 2 and 3 , respectively. It is obvious that each participant can perform modulation operations to obtain a deterministic channel for controlled teleportation. Next, let us further analyze one universal example.

Assume that Alice and Bob share the following quantum state as a channel $[33,34]$ :

$$
\begin{aligned}
|\varphi\rangle_{X_{1} Y_{1} X_{2} Y_{2} \cdots X_{n} Y_{n}}= & \sum_{p_{1}=0}^{p_{1}=1} \sum_{p_{2}=0}^{p_{2}=1} \cdots \\
& \sum_{p_{n}=0}^{p_{n}=1} a_{p_{1}, p_{2}, \cdots p_{n}}\left|p_{1} p_{2} \cdots p_{n}\right\rangle_{X_{1} X_{2} \cdots X_{n}} \\
& \otimes\left|p_{1} p_{2} \cdots p_{n}\right\rangle_{Y_{1} Y_{2} \cdots Y_{n}}
\end{aligned}
$$

where Alice and Bob hold particles $\left\{X_{i}\right\}(i=1,2, \ldots n)$ and $\left\{Y_{i}\right\}$ $(i=1,2, \ldots n)$, respectively. Here, according to the analysis of Section 1 , it can be shown that either $\left\{X_{i}\right\}(i=1,2, \ldots n)$ or $\left\{Y_{i}\right\}$ $(i=1,2, \ldots n)$ can play the role of the particular particle set $\left\{A_{i}\right\}(i=1,2, \ldots n)$.

Next, either Alice or Bob imports an auxiliary qubit, performs the appropriate operation and makes a measurement to adjust the channel.

Finally, if the measurement succeeds, the joint system will be transformed into the following form:

$$
|\varphi\rangle_{X_{1} Y_{1} X_{2} Y_{2} \cdots X_{n} Y_{n}}=\prod_{i=1}^{n}\left(|00\rangle_{X_{i} Y_{i}}+|11\rangle_{X_{i} Y_{i}}\right) \text {. }
$$

This demonstrates that a faithful channel composed of $n$ EPR pairs has been formed between sender and receiver that can be used to teleport the unknown state of a $n$-qubit system.

In the event that the measurement fails, the state of the joint system will be

$$
\begin{aligned}
|\varphi\rangle_{X_{1} Y_{1} X_{2} Y_{2} \cdots X_{n} Y_{n}}= & \sum_{p_{1}, p_{2}, \cdots p_{n}=0}^{1}\left(\sqrt{a_{p_{1} p_{2} \cdots p_{n}}^{2}-a_{m}^{2}}\right. \\
& \left.\left|p_{1} p_{2} \cdots p_{n}\right\rangle_{X_{1} X_{2} \cdots X_{n}}\left|p_{1} p_{2} \cdots p_{n}\right\rangle_{Y_{1} Y_{2} \cdots Y_{n}}\right) .
\end{aligned}
$$

Nevertheless, it can be found that the remaining particles $\left\{X_{i}\right\}(i=1,2, \ldots n)$ and $\left\{Y_{i}\right\}(i=1,2, \ldots n)$ still have the possi- 
bility of forming a deterministic channel if appropriate operations are applied to the joint system. In this way, the joint system comprising particles $\left\{X_{i}\right\}(i=1,2, \ldots n)$ and $\left\{Y_{i}\right\}(i=1$, $2, \ldots n)$ can be reused as an important communication resource rather than be abandoned directly after the initial failed attempt. Therefore, a subsequent attempt can be initiated to establish a faithful channel.

One can see that since either $\left\{X_{i}\right\}(i=1,2, \ldots n)$ or $\left\{Y_{i}\right\}$ $(i=1,2, \ldots n)$ dominates the same position of the particular particle set $\{A\}$ mentioned in Section 2, either the sender or the receiver can perform iterative channel modulations until either one succeeds. Interestingly, an arbitrary choice of high-dimensional gate operator can greatly reduce the implementation complexity of terminal node in a network.

Therefore, when any quantum state obtained from a physical realization is considered as a candidate for a quantum channel, it should first be written in the form of state (1). Next, choose a particular particle set; this particular particle set $\left\{A_{i}\right\}(i=1,2, \ldots n)$ must be kept by one participant to be able to implement local high-dimensional quantum gates during execution of a faithful teleportation protocol. The precious entangled qubit resource can subsequently be reused.

\section{General discussion}

With the proliferation of quantum information results, it is possible to adopt more information technologies to quantum control theory. Since quantum teleportation could be viewed as a feed-forward process and can accomplish some distant quantum feedback control tasks, it can be used to obtain and transmit feedback information. In realizing this generalized teleportation via various channels, we initially presented a scheme for quantum state probabilistic transformations assisted by ancillary particles that furnished the following advantages: (1) it provided a way to identify and group a new particular particle set; and (2) with use of this new particle set, it enabled applications of lower-dimensional local operations that implement the state transformation with a certain probability. Next, based on this scheme, we analyzed the existing probabilistic teleportation scheme and constructed in an elegant and simple manner a general teleportation protocol via various quantum states obtained from the potential physical realization. Finally, we provided some concrete examples to explore the feasibility of our scheme. These results have helped to highlight the role of our scheme and provided new insights into the mechanisms underlying quantum state transformations and quantum remote control.

This work was supported by the National Natural Science Foundation of China (61104002, 60904034 and 61071214), the Natural Science Foundation of Jiangsu Province (BK2011283 and BK2011377) and the Natural Science Foundation of the Jiangsu Higher Education Institutions of China (10KJB510024).
1 Nielsen M A, Chuang I L. Quantum Computation and Quantum Information. Cambridge, UK: Cambridge University Press, 2000

2 Bennett C H, Brassard G, Crépeau C, et al. Teleporting an unknown quantum state via dual classic and Einstein-Podolsky-Rosen channels. Phys Rev Lett, 1993, 70: 1895-1899

3 Cirac J I, Zoller P, Kimble H J, et al. Quantum state transfer and entanglement distribution among distant nodes in a quantum network. Phys Rev Lett, 1997, 78: 3221-3224

4 Lloyd S. Coherent quantum feedback. Phys Rev A, 2000, 62: 022108

5 Dong D Y, Zhang C B, Chen Z H. Information-technology approach to quantum feedback control. Int J Mod Phys B, 2006, 20: 1304-1316

6 Wu R B, Chakrabarti R, Rabitz H. Optimal control theory for continuous-variable quantum gates. Phys Rev A, 2008, 77: 052303

7 Zhang J, Liu Y X, Wu R B, et al. Transition from weak to strong measurements by nonlinear quantum feedback control. Phys Rev A, 2010, 82: 022101

8 Dong D, Petersen I R. Sliding mode control of quantum systems. New J Phys, 2009, 11: 105033

9 Wu R B, Hsieh M A, Rabitz H. Role of controllability in optimizing quantum dynamics. Phys Rev A, 2011, 83: 062306

10 Zhang J, Wu R B, Li C W, et al. Protecting coherence and entanglement by quantum feedback controls. IEEE Trans Automatic Control, 2010, 55: 619-633

11 Dong D, Chen C, Li H, et al. Quantum reinforcement learning. IEEE Trans Syst Man Cybernetics B, 2008, 38: 1207-1220

12 Gao W B, Goebel A M, Lu C Y, et al. Teleportation-based realization of an optical quantum two-qubit entangling gate. Proc Natl Acad Sci USA, 2010, 107: 20869-20874

13 Gottesman D, Chuang I L. Demonstrating the viability of universal quantum computation using teleportation and single-qubit operations. Nature, 1999, 402: 390-393

14 Kimble H J. The quantum internet. Nature, 2008, 453: 1023-1030

15 Bouwmeester D, Pan J W, Mattle K, et al. Experimental quantum teleportation. Nature, 1997, 390: 575-579

16 Chen W X, Tian X L, Hu M L. Network controlled teleportation of N-qubit state. J Mod Opt, 2010, 57: 1619-1623

17 Salemian S, Mohammadnejad S. An error-free protocol for quantum entanglement distribution in long-distance quantum communication. Chin Sci Bull, 2011, 56: 618-625

18 Yan T, Yan F L. Quantum key distribution using four-level particles. Chin Sci Bull, 2011, 56: 24-28

19 Zhang J F, Long G L, Deng Z W, et al. Nuclear magnetic resonance implementation of a quantum clock synchronization algorithm. Phys Rev A, 2004, 70: 062322

20 Zhang J F, Long G L, Zhang W, et al. Simulation of Heisenberg $\mathrm{XY}$-interactions and realization of a perfect state transfer in spin chains using liquid nuclear magnetic resonance. Phys Rev A, 2005, 72: 012331

21 Zhang Y, Cao W C, Long G L. Creation of multipartite entanglement and entanglement transfer via Heisenberg interaction. Chin Phys Lett, 2005, 22: 2143-2146

22 Wang W Y, Wang C, Zhang G Y, et al. Arbitrarily long distance quantum communication using inspection and power insertion. Chin Sci Bull, 2009, 54: 158-162

23 Long G L, Deng F G, Wang C, et al. Quantum secure direct communication and deterministic secure quantum communication. Front Phys China, 2007, 2: 251-272

24 Huang Y C, Liu M, Suo M. N-particle general W state and probabilistic teleportation. Int J Mod Phys B, 2007, 21: 4387

25 Humble T S, Bennink R S, Grice W P. Simultaneous teleportation of multiple single-photon degrees of freedom. J Mod Opt, 2011, 58: 288-298

26 Deng F G, Li X H, Li C Y, et al. Multiparty quantum-state sharing of an arbitrary two-particle state with Einstein-Podolsky-Rosen pairs. Phys Rev A, 2005, 72: 044301

27 Deng F G, Li X H, Li C Y, et al. Quantum state sharing of an arbitrary two-qubit state with two-photon entanglements and Bell-state measurements. Eur Phys J D, 2006, 39: 459-464

28 Wang Y H, Song H S. Preparation of multi-atom specially entangled 
W-class state and splitting quantum information. Chin Sci Bull, 2008, 54: $158-162$

29 Gao T, Yan F L, Wang Z X. Quantum logic networks for probabilistic teleportation of many particle state of general form. Quant Inform Comput, 2004, 4: 186-195

30 Yan F L, Ding H W. Probabilistic teleportation of an unknown two-particle state with a four-particle pure entangled state and positive operator valued measure. Chin Phys Lett, 2006, 23: 17

31 Liu D M, Wang Y W, Jiang X M, et al. A criterion for quantum teleportation of an arbitrary $\mathrm{N}$-particle state via a $2 \mathrm{~N}$-particle quantum channel. Chin Phys B, 2010, 19: 020307

32 Jiang M, Li H, Zhang $\mathrm{Z} \mathrm{K}$, et al. Faithful teleportation of multi-particle states involving multi spatially remote agents via probabilistic channels. Physica A, 2011, 390: 760-768

33 Jiang M, Li H, Zhang Z K, et, al. Faithful teleportation via multi-particle quantum states in a network with many agents. Quant Inform Proc, 2012, 11: 23-40
34 Yan F L, Wang D. Probabilistic and controlled teleportation of unknown quantum states. Phys Lett A, 2003, 316: 297-303

35 Yang C P, Chu S I, Han S. Efficient many-party controlled teleportation of multi-qubit quantum information via entanglement. Phys Rev A, 2004, 70: 022329

36 Zhang L H, Yang M, Cao Z L. Entanglement concentration for unknown W class states. Physica A, 2007, 374: 611-616

37 Li L Z, Qiu D W. The states of W-class as shared resources for perfect teleportation and superdense coding. J Phys A: Math Theor, 2007, 40: 10871

38 Jiang W X, Fang J Z, Zhu S Q, et al. Probabilistic controlled teleportation of a triplet W state. Chin Phys Lett, 2007, 24: 1144-1146

39 Yan F L, Yan T. Probabilistic teleportation via a non-maximally entangled GHZ state. Chin Sci Bull, 2010, 55: 902-906

40 Dong L, Xiu X M, Gao Y J, et al. Controlled three-party communication using GHZ-like state and imperfect Bell-state measurement. Opt Commun, 2011, 284: 905-908

Open Access This article is distributed under the terms of the Creative Commons Attribution License which permits any use, distribution, and reproduction in any medium, provided the original author(s) and source are credited. 